\title{
Contribution de Daniel Duband à l'hydrométéorologie opérationnelle d'aujourd'hui
}

\author{
Patrick TOURASSE
}

Chargé de Mission Coordination de l'Eau, EDF-DPIH, Lyon-Paris, France

\begin{abstract}
RÉSUMÉ. - Au cours des 50 années qu'il a consacrées à sa carrière, comme statisticien et ingénieur expert au sein d'Electricité de France de 1962 à 1996, puis comme expert conseil indépendant de 1996 à 2011, Daniel Duband a non seulement été à l'origine d'avancées scientifiques de tout premier ordre en hydrologie statistique mais il a aussi mis toute son énergie et son talent d'ingénieur au développement et à la pérennisation de réseaux de mesure hydroclimatologiques représentatifs, à la défense et à la sauvegarde des longues séries de données anciennes et au rapprochement de l'hydrologie et de la météorologie pour qu'émerge, à EDF et en France, une hydrométéorologie opérationnelle de qualité. Sans se vouloir exhaustive de tous les apports de D. Duband à l'hydrométéorologie d'aujourd'hui, cette communication est un hommage à toute l'étendue de sa contribution et à la valeur des principes qu'il a su transmettre à plusieurs générations d'ingénieurs et de techniciens.
\end{abstract}

Mots-clés : D. Duband, réseaux de mesure, données anciennes, prévision hydrologique, hydrométéorologie opérationnelle.

\section{Daniel Duband contribution to EDF and French modern operational hydrometeorology}

\begin{abstract}
During the 50 years of his career, first as statistician and senior hydrologist at Electricite de France from 1962 to 1996, then as freelance consulting expert from 1996 to 2011, Daniel Duband not only brought high level scientific advances to statistical hydrology but also put all his talent to develop representative and permanent hydroclimatological networks, to save long series of hydro-climatological observations and to promote modern operational hydrometeorology towards helping putting together both meteorological and hydrological communities.

Not being exhaustive of all the hydro-meteorological contribution of D. Duband, this paper is a tribute to the wideness of main technical advances and values he succeeded to transmit to generations of EDF and French engineers and technicians.
\end{abstract}

Key-words: D. Duband, hydrological networks, Data rescue, hydrological forecasting, operational hydrometeorology.

\section{INTRODUCTION}

Comme le rappelle Ch. Obled (2013), dans l'hommage qu'il consacre à l'importante contribution scientifique de Daniel Duband à l'hydrologie des cinquante dernières années, les méthodes d'analyses statistiques et les outils de prévision hydrologique que D. Duband a su développer et mettre en application au cours de sa longue carrière sont non seulement nombreux et de grande portée scientifique, mais ils ont aussi accompagné l'émergence puis la consolidation de l'hydrométéorologie opérationnelle d'aujourd'hui.

Que ce soit pour la conception et le dimensionnement des aménagements d'EDF puis pour l'optimisation et la sécurité de leur exploitation, très nombreux sont les ingénieurs et les techniciens de la DTG ${ }^{1}$ d'EDF qui ont eu et qui continuent à produire des études, des analyses et des prévisions en utilisant les outils et les approches statistiques qu'a développés et promus D. Duband pendant toute sa carrière :

- Au premier chef, la méthode du Gradex qui a servi au dimensionnement des évacuateurs de crue de tous les grands barrages de France métropolitaine (Duband et al., 1988)

1. Créée dès 1947 à la nationalisation d'EDF, la Division Technique Générale, plus connue aujourd'hui sous son acronyme DTG est une unité d'expertise spécialisée, au sein d'EDF, dans la mesure et le diagnostic pour les besoins et les enjeux des parcs de production, de transport et de distribution d'électricité. mais aussi, sous d'autres cieux et sous d'autres climats, pour qualifier aux crues les barrages de la Réunion, le complexe de Petit Saut en Guyane (Garçon, 1988) ou le barrage de Yaté en Nouvelle-Calédonie (Tourasse, 1991).

- Egalement pour de nombreuses expertises ou contre-expertises d'EDF dans des contrées où l'hydrologie anglo-saxonne et les approches maximisantes de la PMP-PMF² ont généralement meilleur droit de cité que l'approche probabiliste de l'école française : au Canada (Duband, 1994), en Inde, au Brésil et même en Chine.

- Plus récemment, dès le début des années 2000, pour servir de base à la méthode SCHADEX (Paquet et al., 2013) qui est maintenant utilisée en référence par la DTG pour le dimensionnement et la vérification des évacuateurs de crues des grands barrages EDF. Cette nouvelle méthode qui corrige certaines faiblesses de la méthode du Gradex (Duband et Garçon, 1992) reprend les grands principes de la méthode du Gradex mais approche la loi des précipitations extrêmes par une analyse par type de temps et la distribution des débits extrêmes via un processus de Monte-Carlo croisé à une modélisation hydrologique à réservoirs.

- Bien sûr, aussi, la méthode des Analogues (Duband, 1970) qui a permis à EDF de produire, dès la fin des années soixante, de premières prévisions quantitatives de

2. PMP - PMF : Probable Maximum Precipitation - Probable Maximum Flood 
précipitation journalières en montagne à l'époque où les modèles numériques de la Météorologie Nationale - parce que construits avec des résolutions bien trop larges sur les zones de relief - ne parvenaient pas à approcher ces prévisions avec une précision suffisante.

- Méthode qui est largement et efficacement utilisée aujourd'hui en complément des modèles physiques de Météo-France et qui, comme le souligne Ch. Obled (2013), a fait l'objet de nombreux travaux récents de développement et de recherches, notamment universitaires, pour améliorer la performance de son moteur d'analogie (S. Guilbaud, 1997 ; Bontron 2004) ou pour chercher dans des descripteurs plus fins de la situation météorologique et de son évolution prévue, des prédicteurs plus adaptés à la prévision quantitative des précipitations (Ben Daoud, 2010 ; Horton, 2012).

- Egalement, le recours constant à la statistique multi-linéaire et aux corrélations multiples pour mettre en œuvre et améliorer, au fil des années : la représentativité des réseaux de mesure (Duband, 1979); la prévision des apports de remplissage des grands réservoirs EDF; le calcul de la probabilité des étiages sévères et la prévision des étiages des grands cours d'eau notamment de la Loire (Duband, 1970) ; enfin et surtout, dès la fin des années soixante dix, la prévision des crues des petits bassins de montagne par l'approche originale de la Différence Première de la Fonction de Transfert (DPFT) qui reprend le concept classique de «1'hydrogramme unitaire » mais qui, sans imposer de modèle a priori à la "production » des volumes écoulés, approche celle-ci par un processus itératif et convergent de convolution-déconvolution (Duband et al., 1993) qui ne pouvait naître que dans l'esprit très éclairé d'un hydrologue-statisticien comme D. Duband.

Au-delà des outils et des méthodes que $\mathrm{D}$. Duband a développés et promus au cours des 35 années qu'il a passées à EDF, nombreux sont ceux - j'en fais partie bien sûr qui ont cheminé professionnellement en s'appuyant sur les grandes compétences et l'expérience de D. Duband et qui se sont forgés à ses exigences de qualité et de rigueur mais aussi à sa pugnacité et, parfois, à son intransigeance.

Plus qu'un scientifique ou un statisticien, D. Duband était aussi un grand technicien doté d'un vrai sens physique et d'une excellente mémoire des évènements hydrologiques du passé. Les étiages de la Loire n'avaient aucun secret pour lui, ni d'ailleurs toutes les grandes crues de ce fleuve qu'il tenait en référence pour la longueur et la qualité de ses longues séries d'observation (Duband et al., 2004). Il avait aussi une parfaite connaissance de tout l'historique de nos cours d'eau et se plaisait toujours à rappeler avec une précision d'horloger les débits ou les pluies qui avaient été observés lors de tel ou tel évènement de crue dans les Alpes, les Pyrénées ou le Massif-Central. Bien malin celui qui savait le prendre en défaut à ce sujet.

Grand spécialiste de son domaine, expert en hydrométéorologie opérationnelle dont il accompagna et fit grandir le concept au sein d'EDF avant de le promouvoir dans les nombreuses instances scientifiques et institutionnelles auxquelles il a toujours participé, D. Duband a contribué de manière décisive au rapprochement des communautés de météorologues et d'hydrologues en France. Il a aussi mis toute son énergie et son talent au développement et à la pérennisation de réseaux de mesure hydro-climatologiques représentatifs, à la défense et à la sauvegarde des longues séries de données anciennes pour qu'émerge, à EDF et en France, une hydrométéorologie opérationnelle de qualité.
Sans prétendre à l'exhaustivité, c'est ce que nous allons essayer de balayer. Le document qui suit se veut d'abord un hommage à un grand ingénieur et à un homme qui a beaucoup apporté à l'hydrologie et à qui plusieurs instances ont déjà tenu à souligner la trace féconde qu'il a laissée.

C'est aussi un complément à l'hommage scientifique que Ch. Obled (2013) lui rend à l'occasion de ce colloque SHF sur les " événements extrêmes d'inondation ». Colloque qui fait suite au premier que D. Duband avait organisé en mars 2006 à Lyon et au suivant, de février 2012, dont il avait commencé à bâtir le programme avant de nous quitter, le 5 juillet 2011.

Par la confiance qu'il m'a toujours accordée, je lui dois personnellement tout ce que j'ai appris de mon métier. J'ai l'occasion, ici, de le remercier. Je le fais avec bonheur et simplicité. Pour autant, comme l'écrit Ch. Obled (2013) dans son propos introductif « l'exercice est redoutable » tant la contribution de D. Duband est vaste et l'impression d'en laisser de côté, toujours présente.

\section{DE LA STATISTIQUE HYDROLOGIQUE À L'HYDROMÉTÉOROLOGIE OPÉRATIONNELLE D'AUJOURD'HUI}

Comme déjà vu, c'est la prévision des apports aux grands réservoirs EDF puis la prédétermination des crues extrêmes pour la vérification et la conception des évacuateurs de crues existants ou à construire, qui ont très vite mobilisé le statisticien qu'était Daniel Duband lorsqu'il entre à EDF au début des années soixante.

A cette époque, les données d'observation sont encore rares en montagne. Malgré l'effort d'équipement en stations de mesure hydrologiques conduit, dès avant l'aube des années cinquante, par la DTG et les Régions d'équipement d'EDF, la plupart des chroniques de débits et de précipitations disponibles n'ont que quelques années d'existence et la prévision statistique des écoulements et des apports de fusion en montagne n'en est encore qu'à ses débuts.

Avec des outils qui n'ont rien à voir avec la panoplie numérique de l'hydrologue d'aujourd'hui - les calculs se font alors encore souvent à la main (Figure 1) -, il s'agit déjà de traquer les moindres inter-corrélations d'explication qui existent entre les précipitations d'hiver, l'enneigement lorsqu'il est mesuré et les apports de fusion aux réservoirs.

La corrélation simple entre variables mais surtout, très vite, la corrélation multiple et l'interprétation des coefficients de corrélation partielle pour aider au meilleur choix des variables prédictives sont alors de toutes les approches d'une hydrologie statistique prédictive qui se met en place et à laquelle D. Duband apporte rapidement sa meilleure contribution et son esprit critique. Parce que les données sont rares, il faut se garder des pièges des liaisons trop parfaites et des effets d'échantillonnage que ne manquent pas d'engendrer des séries encore trop courtes ou des données parfois encore insuffisamment calibrées.

Par sa formation et son expérience de statisticien, D. Duband excelle à déjouer tous ces pièges. Avec son sens physique et sa connaissance fine de l'hydrologie de tous les cours d'eau de montagne - comme déjà mentionné, il n'est pas un évènement hydrologique de la sécheresse de 1921 à la crue de la Loire du 21 septembre 1980 qui n'ait marqué sa mémoire - il se plaît parfois, non sans quelque malice, à prendre ses interlocuteurs en défaut d'un évènement trop vite oublié ou d'une corrélation trop rapidement interprétée. 


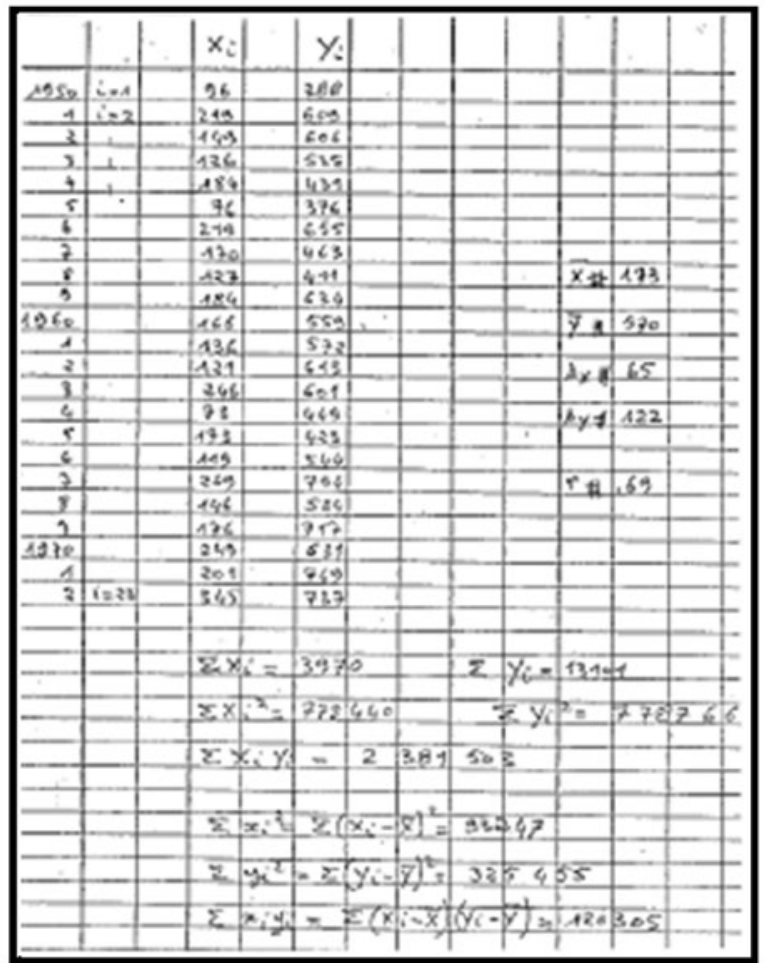

Figure 1 : Calcul manuel des corrélations précipitations-écoulements d'une rivière des Pyrénées - Archives personnelles D. Duband.
De façon constructive et comme en écho au cours d'hydrologie qu'il va tenir pendant de longues années à l'Ecole d'Hydraulique de Grenoble (ENSHG), il transmet aux techniciens et aux ingénieurs qui travaillent à ses côtés toutes les ficelles et tout l'art de l'analyse statistique appliquée à l'hydrologie quantitative et à la prévision.

C'est d'ailleurs parce qu'il attache une très grande importance à la mémoire et à la genèse des situations météorologiques et hydrologiques qui sont à l'origine des évènements marquants du climat, qu'il va mettre en place à EDF et constamment le défendre dans les nombreuses instances et colloques auxquels il participera - une véritable culture du retour d'expérience.

Alimenté par des monographies détaillées de chaque évènement qu'il n'a de cesse de demander aux équipes de mesure et de prévision de la DTG - travail qui continue à être poursuivi, dans le même esprit, par ses successeurs -, cet ensemble de données et de renseignements sur les grands évènements hydrologiques constitue, aujourd'hui, un véritable patrimoine de l'observation du climat passé. C'est aussi, par l'étude des conséquences de ces événements sur l'hydrologie des cours d'eau et sur l'exploitation des aménagements de production d'électricité, un puissant outil d'aide à l'analyse de risques des évènements à venir.

Au-delà de l'importance qu'il accorde à la mémoire hydrologique des évènements et à l'explication de leur genèse par l'étude fine de la relation entre précipitations et débits, D. Duband, qui n'est pas météorologue de formation, comprend très vite aux côtés de P. Guillot que l'hydrologie opérationnelle,

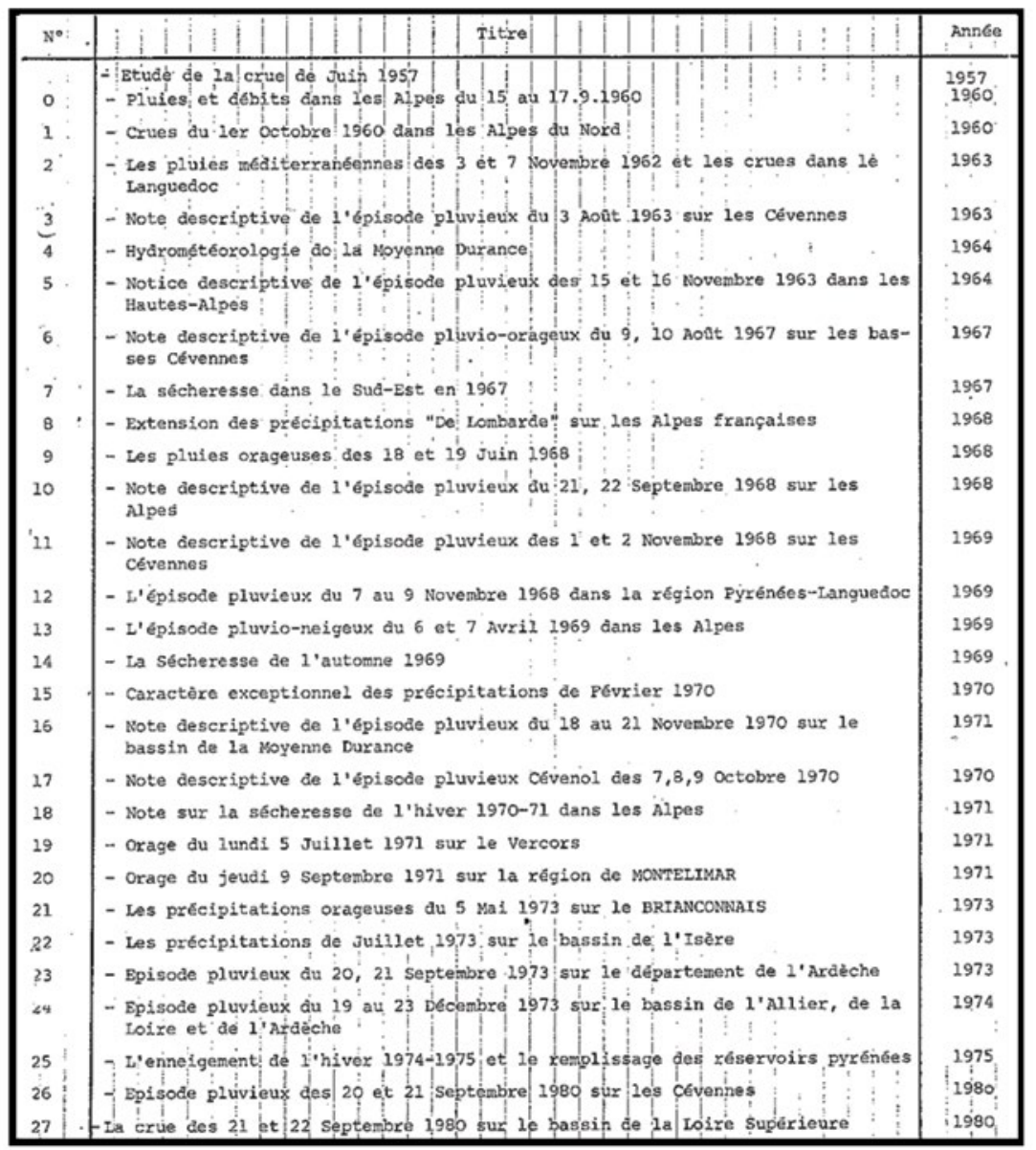

Figure 2 : Liste des monographies DTG d'épisodes pluvieux remarquables entre 1950 et 1980 (Source Documentation DTG et archives personnelles D. Duband). 
fût-elle statistique ou simplement descriptive, ne saurait se développer favorablement sans l'aide de la météorologie.

Même s'il peine alors à voir reconnaître ses travaux par une communauté des météorologues plus adepte de la modélisation physique, il persiste à promouvoir le bien fondé de l'approche statistique pour la prévision quantitative des précipitations en montagne et commence à tisser des liens étroits avec les équipes de recherche et de prévision de la Météorologie Nationale de l'époque.

Ainsi, fort de la densité des réseaux EDF de mesure de précipitations et de leur bonne représentativité en zone de montagne, il facilite alors les échanges de données entre EDF et la Météorologie Nationale pour permettre aux météorologues de mieux calibrer leurs modèles de circulation en zone de relief. En contrepartie, il obtient de la Météorologie Nationale, la mise à disposition conventionnelle des champs de géopotentiels observés et prévus chaque jour ; données qui vont, dès lors, permettre à EDF de produire au quotidien, de façon d'abord semi manuelle puis entièrement automatique, ses propres prévisions quantitatives de précipitations en montagne.

Parallèlement, dès la fin des années soixante, il s'appuie sur les prévisionnistes détachés de la Météorologie Nationale dans les centres de prévision EDF de Lyon pour les Alpes et de Toulouse pour les Pyrénées et le Massif-Central pour améliorer les prévisions de productibilité à courte échéance et permettre aux Centres Régionaux des Mouvements d'Energie d'EDF de mieux optimiser l'utilisation des moyens de production.

Il s'agit alors, pour EDF, de mieux anticiper, par l'analyse classique des cartes météorologiques observées et prévues - c'est l'époque, aujourd'hui révolue, des facs similés à impression thermique qui crachotent, à intervalles réguliers, les cartes d'analyses et les sorties de modèles de la météorologie Nationale - l'arrivée des perturbations pluvieuses qui ont une incidence de court terme sur l'évolution du productible hydraulique. Il s'agit aussi d'agrémenter les prévisions probabilistes de précipitations d'une expertise météorologique qui s'avère vite indispensable à leur qualification pour les usages de la prévision hydrologique en montagne.

De ces dialogues quotidiens entre les prévisionnistes détachés de la Météorologie Nationale et les ingénieurs ou hydrologues d'EDF, du croisement de leurs sensibilité et de leurs compétences respectives, va émerger une complémentarité d'approche que D. Duband ne va cesser de défendre et de promouvoir pendant toute sa carrière.

$\mathrm{Au}$ fil des années, ces ingénieurs de la météorologie vont transmettre aux hydrologues EDF, une culture de la météorologie qui leur permettra petit à petit d'élargir leur champ d'intervention et d'apporter aux centres de décision EDF un appui objectif non seulement pour la prévision des débits (crues, étiages) et des apports aux réservoirs (prévision saisonnière) mais aussi pour la surveillance et l'anticipation de toutes les situations à risques pour l'exploitation du système électrique.

On parle ici, entre autres : des épisodes de neige collante qui peuvent avoir des conséquences dommageables sur les réseaux de transport et de distribution d'électricité; des tempêtes de vent (tempêtes du 26 décembre 1999 et du 24 janvier 2009) ou des situations de canicule qui ont, elles, un impact direct sur la température des cours d'eau et sur l'exploitation des centrales nucléaires de bord de rivière (GT Thermie, 2012).

S'agissant du risque " neige collante », il faut se souvenir des épisodes marquants du 27 décembre 1970 dans la vallée du Rhône, du 11 janvier 1981 dans le Roussillon et du 26 novembre 1982 dans la Haute-Loire, qui eurent tous des conséquences majeures sur les réseaux de distribution et de transport d'électricité ${ }^{3}$.

Dès lors, de nombreuses études furent engagées à EDF pour bien comprendre et décrire le phénomène, pour en cartographier quantitativement le risque (Plancher, 1976 ; Deneau et Guillot, 1984) et mieux cibler les politiques préventives de renforcement des réseaux et, enfin, pour essayer de prévoir et d'anticiper l'occurrence du phénomène.

Pour la prévision, deux approches différentes sont, à l'époque, développées et progressivement mises en œuvre : — une approche plutôt physique, basée sur la modélisation simplifiée des mécanismes d'accrétion de la neige autour des câbles aériens et développée par les équipes de recherche de la R\&D d'EDF en collaboration avec le CNRS (J.-L. Lapeyre, 1990);

- une approche statistique mise au point par D. Duband (1973) avec la collaboration de l'institut de Mathématiques Appliquées de Grenoble (IMAG) et qui va permettre (Duband et Deneau, 1982) de calculer quotidiennement, après avoir croisé le fichier des incidents de neige et de givre constatés sur le réseau de transport d'électricité avec le fichier des situations météorologiques observées et prévues, un macro indicateur " neige collante ».

Cet indicateur de risque, complété par la surveillance temps réel des précipitations et des températures de l'air dans les zones à risque et par le suivi et l'analyse fine des situations météorologiques génératrices, va ensuite montrer toute sa pertinence lors des épisodes majeurs de la fin des années 80 qui suivront, notamment celui qui frappa le nord-Isère du 9 au 12 décembre 1990 (Tourasse et Drouet, 1990).

C'est l'époque où la moindre alerte neige collante voyait les équipes de mesure DTG partir aux fins fonds des Pyrénées, sur les contreforts du Massif-Central ou sur les terres froides du nord Isère, pour mesurer, au cœur de l'épisode et avec des kits de micro sondage spécialement développés à cet effet, la densité de la neige fraîchement tombée au sol ou la dimension et la charge linéaire des manchons en formation. S'en suivaient, des discussions sans fin entre spécialistes pour savoir et décider, de façon définitive, si tel épisode ou telle séquence au cœur d'un épisode pouvait être qualifiée de «collante » ou non! De façon constructive, il s'agissait surtout de savoir par le retour-terrain, si la prévision avait vu juste ou non et si les analyses amont, tant météorologiques que statistiques, correspondaient à la réalité observée sur le terrain.

Là encore, comme évoqué précédemment pour la prévision hydrologique, c'est bien le croisement entre l'analyse fine des dynamiques météorologiques et les outils statistiques sur-mesure développés à la DTG sous l'égide de D. Duband qui va vraiment faire progresser la capacité d'EDF à mieux anticiper ces phénomènes. S'y ajoutait la demande constante de D. Duband à ses équipes, de toujours confirmer par des mesures de terrain, la réalité des chiffres que les modèles de prévision ou les analyses statistiques laissaient entrevoir.

De cette imbrication étroite et permanente entre la météorologie et l'hydrologie, de cette continuité d'analyse des conséquences des précipitations sur les débits ou sur d'autres

3. Le 11 janvier 1981, 5000 poteaux des réseaux basse et moyenne tension et 120 pylônes haute tension ont été totalement détruits privant 300000 personnes d'électricité pendant plusieurs jours. De même, le 27 novembre 1982, ce sont plus de 6000 poteaux basse et moyenne tension et près de 70 pylônes haute tension qui ont été détruit dans la Loire et la Haute-Loire, privant plus de 600000 foyers d'électricité. 


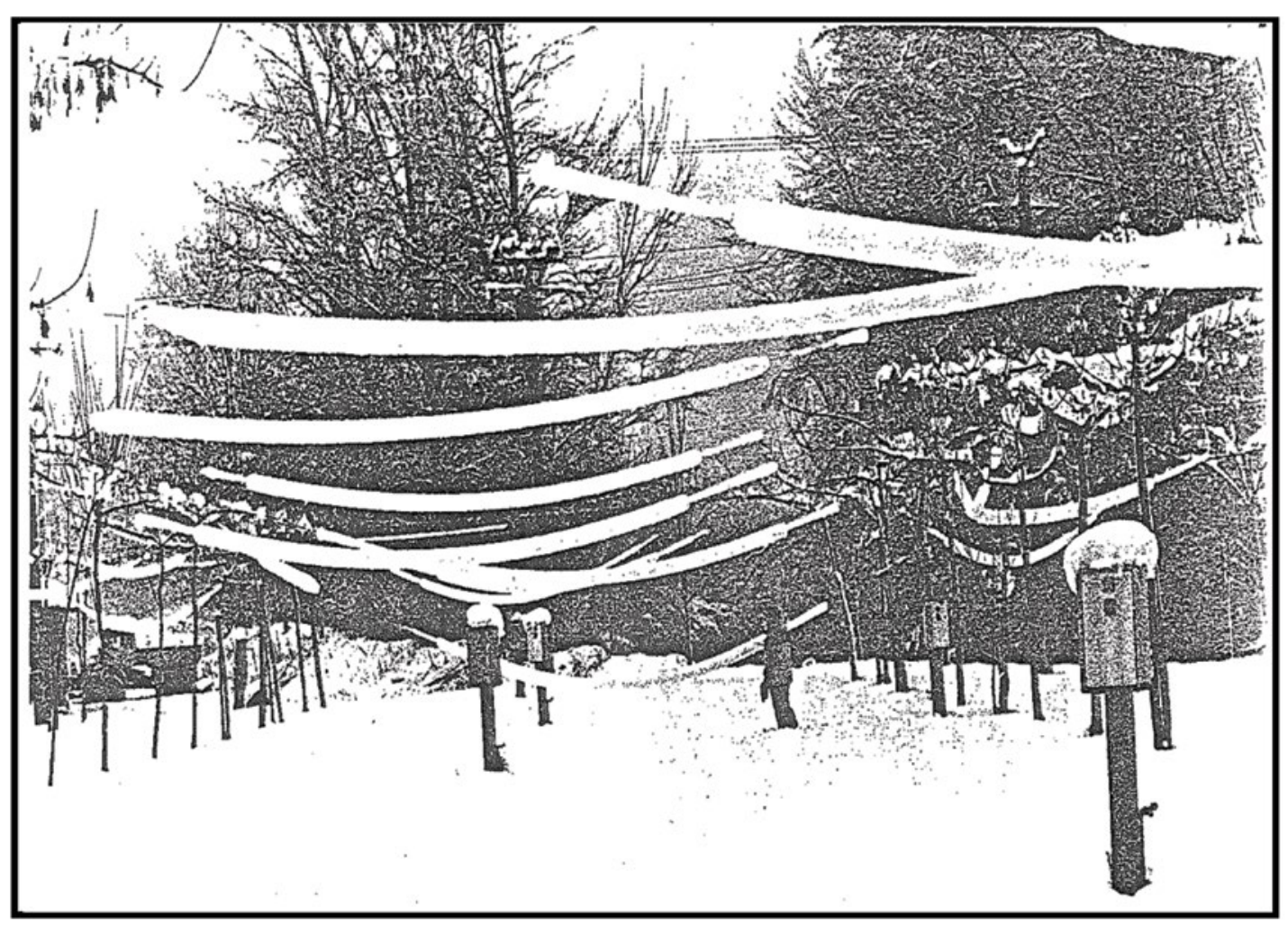

Figure 3 : Manchons de neige collante observés sur fils d'étendage à Barbazan (450m) lors de l'épisode neigeux des 30 et 31 janvier 1986 sur les Pyrénées et le Roussillon (Pinte et Berrut, 1986).

risques, va progressivement se développer à EDF, le concept d'hydrométéorologie opérationnelle qui a trouvé, depuis, d'autres lettres de noblesse - avec notamment la création en 2003 au sein du Ministère de l'Environnement et des Transports du Service Central d'Hydrométéorologie et d'Appui à la Prévision des Inondations (SCHAPI) - mais qui, dans les années 80-90, était encore peu répandu et se voyait même préconisé par certains (Castaing, 1993) comme solution pour prévenir les intempéries et améliorer la prévention des inondations en France.

A EDF, le terme "d'hydrométéorologie », mariage de l'hydrologie et de la météorologie, fait son apparition dès la création en 1947 du Service de la Production hydraulique (SPH) d'EDF (Maurin, 1995). Dès cette époque (1950), la Division Technique Générale du SPH comprend, en effet, un département " hydrométéorologie » réparti entre le site de Grenoble et les premiers Centres Hydrométéorologiques créés en 1947 à Lyon et 1948 à Toulouse.

Plus tard, après la création, en 1969, du service «Energétique et Prévisions » qui reprendra les activités de prévision d'apports aux grands réservoirs et d'hydrologie statistique, puis, en 1975, du « Service Ressources en Eau » qui réunira les équipes de mesures hydrométriques avec les équipes de prévision de la DTG, c'est en 1983, sous l'égide de P. Guillot et D. Duband, que l'hydrométéorologie opérationnelle à EDF va prendre tout son essor, avec la création de deux Centres régionaux de prévision hydrométéorologique opérationnelle : le Centre Hydrométéorologique Alpes (CHA) à Grenoble que D. Duband dirigera de 1983 à 1985 et le Centre Hydrométéorologique Pyrénées-Massif Central (CHPMC) qui sera implanté à Toulouse ; lesquels dotés maintenant de moyens humains et techniques modernisés et renforcés sont toujours en opération aujourd'hui.
Cette création des deux centres hydrométéorologiques Alpes et Pyrénées-Massif-Central fut, de plus, l'occasion de réunir sous un même management les équipes de mesure hydrométriques et les équipes de prévision - historiquement ces deux métiers s'étaient développés au sein de DTG dans des services différents - pour que chacune des activités se nourrisse des besoins et des contraintes mais aussi des richesses de l'autre et que s'estompe une dichotomie historique qui avait longtemps existée entre spécialistes de la mesure d'un côté et prévisionnistes de l'autre.

C'est à cette époque également, au début des années 80 , que l'automatisation de la collecte des données hydrologiques prend tout son essor à EDF. D'abord sur le Rhône avec la mise en œuvre du Système de Prévision Hydrologique du Rhône (SPHYR) puis sur l'ensemble des bassins surveillés par la DTG, avec le déploiement progressif du système $\mathrm{SCHTROUMPH}^{4}$ qui utilise le réseau téléphonique commuté pour le rapatriement des données en temps réel sur Toulouse, Brive et Grenoble.

Entièrement conçu sous cahier des charges EDF avec des stations mono-cartes qui devaient en faciliter la maintenance, ce système souffre au début de défauts de jeunesse et de taux de défaillance inacceptables sous forte activité orageuse (Perret, 1992) que D. Duband va s'attacher à faire corriger avec l'énergie que l'on sait.

De son côté, l'expérience du Rhône s'avère très riche d'enseignements pour la suite. C'est la première fois, en effet, que des outils de collecte automatique des paramètres climatiques (pluies, neige, température) et hydrologiques (débits) et des

4. SCHTROUMPH : Système de Collecte Horaire en Temps Réel sur Ordinateur Utilisé pour la Prévision de l'Hydraulicité 
modèles statistiques de prévision hydrologique (pluie-débit sur les affluents et propagation le long du Rhône) sont implantés sur des calculateurs d'exploitation pour alimenter, en prévisions temps réel, les deux centres de conduite hydraulique du Rhône à Génissiat et à Châteauneuf.

Ceci, sans filtrage ni expertise des résultats des modèles par des spécialistes de la prévision. Il en résulte une difficulté d'appropriation de l'outil, sans doute trop novateur à l'époque, par les exploitants EDF qui forts déjà d'une bonne connaissance des débits de leurs aménagements ont du mal à voir l'intérêt de ces modèles de prévision qu'ils voudraient plus précis et dont ils peinent, alors, à comprendre toutes les incertitudes.

Dans la modélisation hydrologique temps réel, il faut compter, en effet, non seulement sur les imprécisions des données d'entrée - qu'elles soient purement métrologiques ou liées à des défauts de représentativité intrinsèque des stations de mesure -, sur la capacité du modèle lui-même à bien représenter la complexité du processus de transformation de la pluie en débit mais aussi - cela fut une vraie source de difficultés sur le système Rhône au début - sur la difficulté à prendre en compte, dans la modélisation, la perte d'information liée à des absences de données temps réel (sur défaut d'acquisition ou de transmission principalement).

De cela naîtra progressivement la conviction, continument défendue par D. Duband et aujourd'hui admise par tous, que la prévision hydrologique ou hydrométéorologique opérationnelle ne saurait être qu'affaire de capteurs, d'outils ou de systèmes automatiques - fussent-ils « intelligents » - mais qu'elle doit toujours s'appuyer sur l'expertise humaine pour son élaboration et avant toute diffusion (Garçon et al., 2008).

Progressivement va ainsi s'imposer à EDF, le fait que la prévision hydrométéorologique opérationnelle, pour les bassins à enjeux et à court temps de réponse, doit allier en permanence :

- Des réseaux de mesure fiables et de qualité avec des systèmes d'acquisition temps réel des données tout aussi fiables surtout sous orage et sous conditions météorologiques fortement perturbées.

- Des modèles hydrologiques simples et robustes, qu'ils soient, à EDF, de nature plutôt statistiques comme la DPFT ${ }^{5}$ (Duband et al. , 1993) ou d'inspiration plus physique comme le modèle MORDOR qui sera développé plus tardivement à la DTG par R. Garçon (1996).

— Des outils d'aide à l'analyse et à la prévision météorologiques (sorties de modèles météorologiques à courte et moyenne échéances, imagerie satellitale, mosaïque radar, impacts de foudre, ...) directement accessibles par les prévisionnistes hydrologues.

- Des prévisionnistes experts qui allient une double compétence en météorologie et hydrologie et qui sont capables d'analyser, d'interpréter voire d'adapter et surtout d'expliquer les résultats de la prévision aux interlocuteurs EDF seuls à même, in fine, à décider des options à prendre pour la gestion des aménagements.

Ce modèle intégré de la prévision hydrométéorologique qui est loin, à l'époque, d'exister par ailleurs, sera largement mis en avant par EDF dans les nombreux colloques, groupes de travail, ateliers et commissions qui vont se mettent en

5. DPFT : Différence Première de la Fonction de Transfert moyenne d'un bassin versant qui permet, à chaque pas de temps, d'étaler dans le temps et de transformer en écoulement toute pluie efficace unitaire qui affecte le bassin (Guillot et Duband, 1980). place en France dans les années 80-90 notamment après les épisodes catastrophiques du Grand-Bornand (1987), de Nîmes (1988) et de Vaison-la-Romaine (1992).

D. Duband y apportera toujours une contribution très active et ses avis, parfois tranchés mais toujours teintés d'expérience, participeront considérablement à faire émerger d'abord à EDF et plus généralement en France, une hydrométéorologie opérationnelle de qualité et au service des enjeux de la prévision d'aujourd'hui.

$\mathrm{Nul}$ doute que de vastes progrès sont encore à réaliser, tant la prévision hydrologique sous nos climats, en particulier en zone de montagne et sous influence méditerranéenne, reste difficile. Mais c'est une évidence pour chacun que D. Duband a largement contribué à la structurer et à la faire évoluer vers ce qu'elle est devenue aujourd'hui.

A ce propos, il convient de souligner aussi le rôle important qu'a joué D. Duband dans la prise en compte de l'environnement dans la surveillance et la prévision hydrologique. Si cette dimension était peu présente jusqu'au début des années 70 , D. Duband et la DTG comprennent très vite au moment du démarrage du programme nucléaire, qu'il y a là un enjeu majeur pour EDF. Il s'en suit la mise en place du réseau EDF de surveillance des températures amont des sites nucléaires qui fera vite référence et qui va permettre de mieux comprendre le fonctionnement thermique des grands cours d'eau (Poirel, 1997 ; Etude Thermique Rhône, 2006). Puis, au fil des années, le développement progressif de la prévision environnementale - notamment des températures de l'eau - qui prend son plein essor après la canicule de 2003 et qui est maintenant une composante importante de l'expertise DTG.

\section{LA DÉFENSE DES RÉSEAUX PERMANENTS DE MESURE HYDRO-CLIMATOLOGIQUES}

En vrai statisticien et fin connaisseur de l'importance de la qualité des données d'entrée dans toute approche prédictive, D. Duband comprend très vite toute l'importance qu'il faut accorder à la représentativité des stations de mesure et à la qualité des données qu'elles permettent d'acquérir.

Même si, au départ, comme mentionné supra, les activités de prévision et d'observation climatologique (précipitations, températures) d'EDF et les activités de bilans en eau et de mesures hydrométriques se sont développées dans des entités séparées à la DTG - on a vu précédemment que ces activités n'ont été réunis qu'en 1975 à la création du Service Ressources en eau puis mises sous un management commun en 1983 avec la création des centres hydrométérologiques régionaux d'EDF -, D. Duband qui rejoint P. Guillot au siège de la DTG à Grenoble en 1966, participe très tôt aux réflexions sur la bonne représentativité des réseaux de mesure d'EDF.

A cette époque, les réseaux hydrologiques et climatologiques en zone de montagne sont encore en construction. La Météorologie Nationale est encore peu impliquée dans l'observation de la climatologie de montagne - elle s'y engagera davantage après l'avalanche meurtrière de Val d'Isère en 1970 et le développement du Centre d'Etude la Neige (CEN) pour la prévention du risque d'avalanches sur tous les massifs - et les besoins EDF pour accompagner le développement de l'hydroélectricité en France, sont immenses.

Ainsi, de quelque déjà 600 stations de mesures EDF en 1950 , le réseau d'observations des précipitations en montagne 
passera à près de 1200 stations à la fin des années 60 (Figure 4). Même évolution pour les stations manuelles de mesure de l'enneigement - les perches à neige - dont le nombre culminera à plus de 600 points de mesure à la fin des années 70 (encart Figure 4, ci-après).

Pendant ces périodes de constitution - puis au milieu des années 70 de rationalisation - des réseaux climatologigues d'EDF, D. Duband, fort de sa compétence statistique en analyse spatiale des données et en étude des séries chronologiques, apporte tout son savoir-faire et la pertinence des outils d'analyse multilinéaire qu'il développe (Corrélation multiple, Analyse en composantes principales (ACP), ...) pour aider à choisir les emplacements de stations climatologiques les plus représentatifs et les plus fiables.

Il participe ainsi, dans les années 70, à la mission EDF de modernisation des réseaux de données hydrologiques, dite Mission Anglaret, du nom de L. Anglaret, chargé de mission près du Chef du Service de la Production Hydraulique (SPH), en charge de la consistance de cette modernisation. Lancée pour rationaliser et automatiser la collecte des données d'EDF et pour en concentrer les enjeux sur les besoins du parc hydraulique en exploitation - à la fin des années 70, le parc nucléaire est en plein développement -, la commission Anglaret va aboutir à une diminution de 2/3 des réseaux climatologiques EDF et de $50 \%$ du réseau des perches à neige.

Pour autant, convaincus qu'une série chronologique, même courte, de données observées constitue déjà un capital irremplaçable d'information, P. Guillot et D. Duband vont tout faire à l'époque pour que les séries existantes ne s'arrêtent pas de fonctionner et qu'elles puissent être reprises, pour les plus représentatives d'entre elles, par les services de la Météorologie Nationale.
Plus tard, dans les années 80 , lorsque se mettra en place, en France, la banque nationale des données pluviométriques dite banque PLUVIO et qu'il s'agira de bien étiqueter la provenance des données sources, D. Duband s'investira alors dans de longues discussions avec la Météorologie Nationale puis avec Météo-France, pour que les données initialement produites par EDF soient bien identifiées comme telles.

C'est l'époque aussi, au début des années 80 , où s'engage à EDF l'accélération de l'automatisation du réseau d'enneigement, avec le développement des télénivomètres à faisceau horizontal mobile ou vertical fixe. Il s'agit de permettre le suivi quotidien de la valeur en eau du manteau neigeux et son actualisation au plus court pour l'optimisation de la gestion des apports aux réservoirs et du productible hydraulique (Duband, 1988). En même temps, suite à la rationalisation du réseau des perches à neige, de concentrer les efforts de surveillance sur les bassins à plus forts enjeux et sur les séries d'enneigement à fort potentiel d'explication pour les volumes de fusion.

Encore fallait-il choisir les points les plus représentatifs pour installer aux meilleurs endroits ces appareils coûteux et donc nécessairement en nombre limité6. Là encore, les méthodes statistique d'analyse spatiale - notamment l'Analyse en composantes principales - que D. Duband avait introduites à la DTG, vont s'avérer très utiles (Figure 5).

L'intérêt de D. Duband pour l'observation de la neige et pour la prise en compte de la mesure de son équivalent en eau dans les modèles de prévision d'apports, a été perma-

6. A l'aube des années 2000, le réseau de mesure d'enneigement d'EDF comprend 36 télénivomètres en France : 9 dans les Pyrénées, 5 dans le Massif-Central, 2 dans le Jura et 20 dans les Alpes.

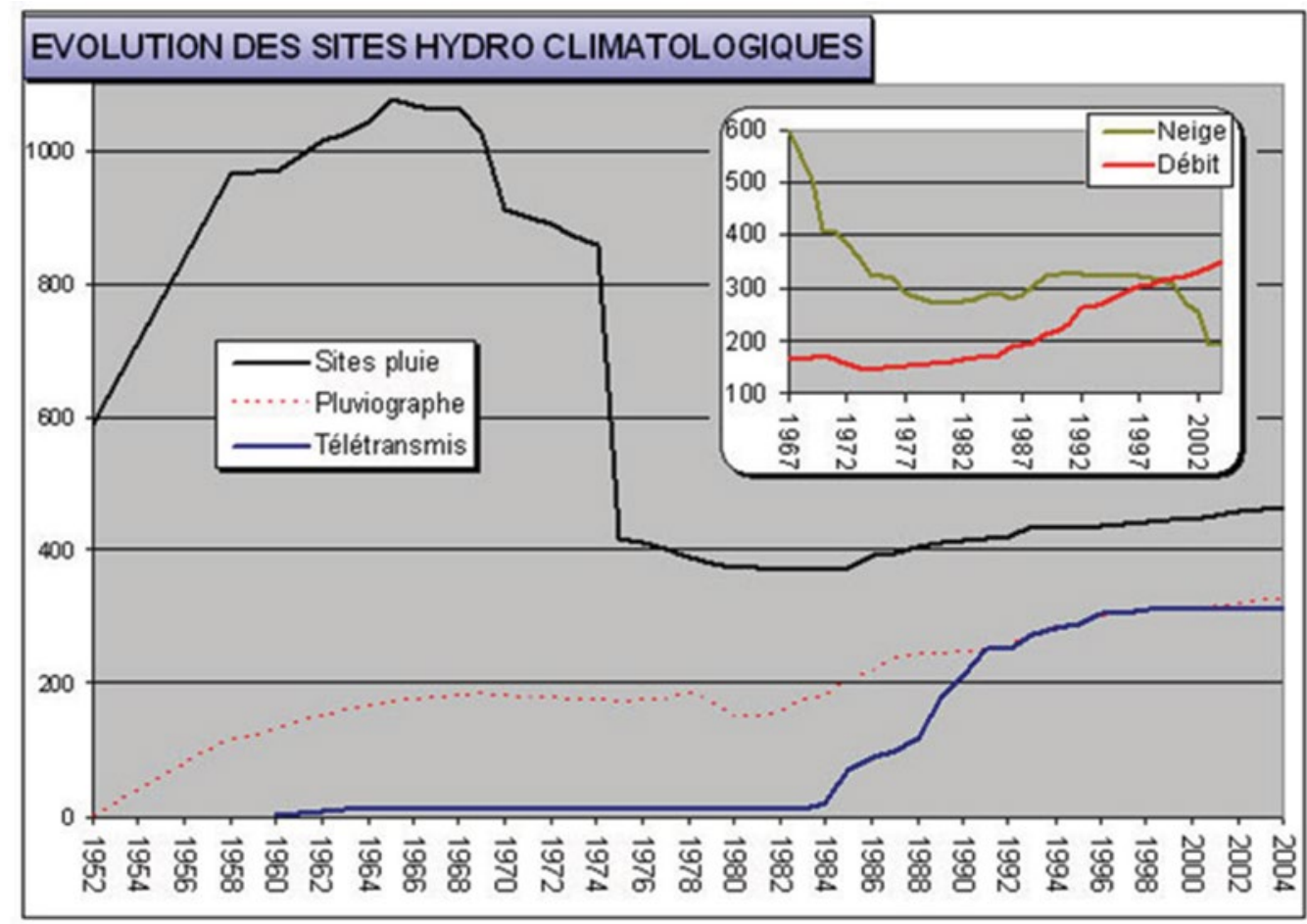

Figure 4 : Evolution du nombre de stations pluviométriques EDF depuis le début des années cinquante. On remarque l'effort engagé dès la nationalisation pour constituer un réseau dense d'observation des précipitations au service du développement de l'hydroélectricité. Egalement, la forte réduction de moyens qui découle, au milieu des années 70, de l'adaptation des réseaux d'observation à l'exploitation du parc hydraulique existant. 


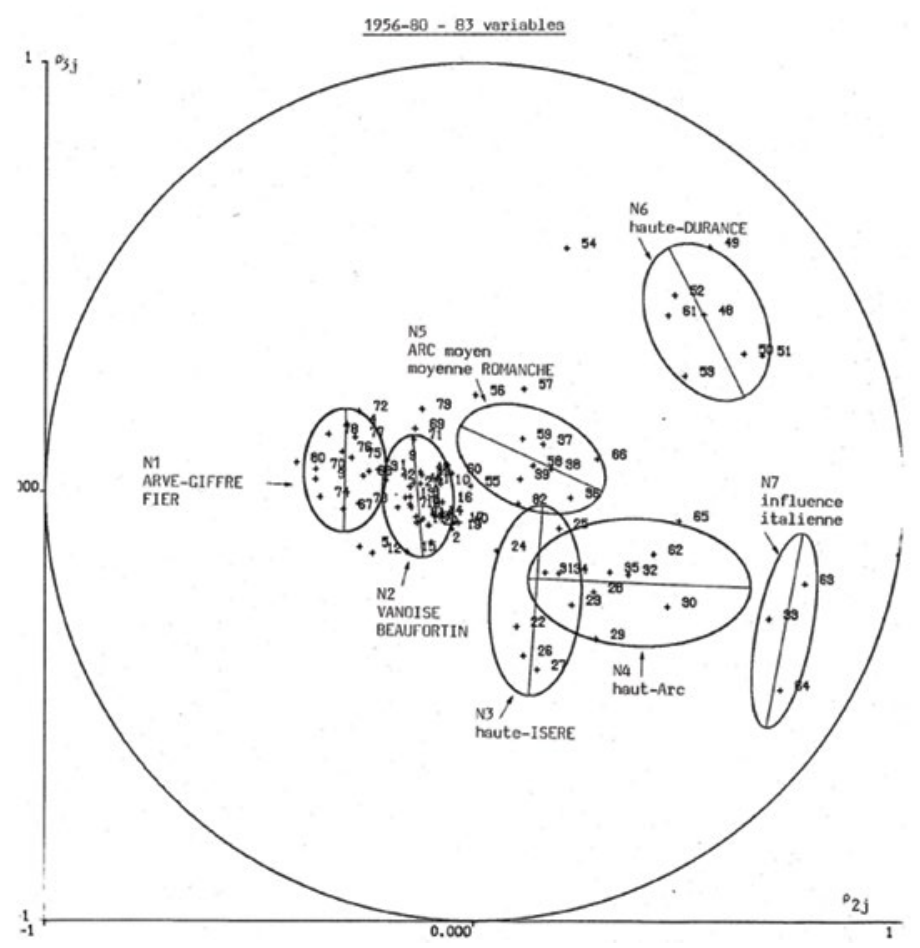

Figure 5: Typologie de l'enneigement des Alpes du Nord par Analyse en composantes principales (ACP) des chroniques d'enneigement de 150 perches à neige observées entre 1964 et 1980 (Pinte, 1981).

nent pendant toute sa carrière. Malgré certaines insuffisances de la mesure ponctuelle de l'enneigement, qu'il reconnaissait explicitement - notamment lorsque la fusion est déjà bien engagée et que le manteau a perdu de son homogénéité spatiale -, il a toujours affiché sa préférence pour la mesure directe de la valeur en eau de la neige par rapport aux approches numériques de modélisation physique du manteau.

Il ne croyait aussi que modérément à la télédétection spatiale pour le suivi opérationnel de l'enneigement ou des surfaces enneigées sur le relief plutôt chahuté de nos massifs. Il favorisa néanmoins certaines expérimentations d'utilisation de l'imagerie satellitale notamment sur les massifs à faibles pentes comme le Massif-Central où la télédétection spatiale pouvait s'avérer un complément utile à la seule mesure sol (Deneau, 1981).

Sa constante était la « mesure » - rien que la mesure - à condition qu'elle soit de qualité et qu'on s'attache, une fois la donnée acquise, à toujours la critiquer et à la valider. Comme il se plaisait à le dire " l'automatisation de la collecte des données en hydrologie, ne sert à rien si on ne s'attache pas, à chaque donnée acquise, à la vérifier et à la contrôler avant de l'archiver dans les bases de données historiques ».

En ce sens, il a été l'un des plus ardents défenseurs de réseaux d'observation hydrologique et hydrométéorologique pérennes et de qualité.

En premier lieu au sein d'EDF, où des logiques de court terme ont pu, à certains moments, remettre en cause la permanence des moyens dédiés à la maintenance des réseaux et à la validation des données. Avec la pugnacité que l'on sait, il se dépense alors sans compter pour convaincre jusqu'aux plus hauts niveaux de l'Entreprise que la qualité des données a un coût mais que ce dernier est sans commune mesure au regard des enjeux de la prévision. Il participe ainsi à maintenir les réseaux de surveillance EDF dans un standard de qualité et de représentativité qui fait encore référence aujourd'hui (Figure 6).

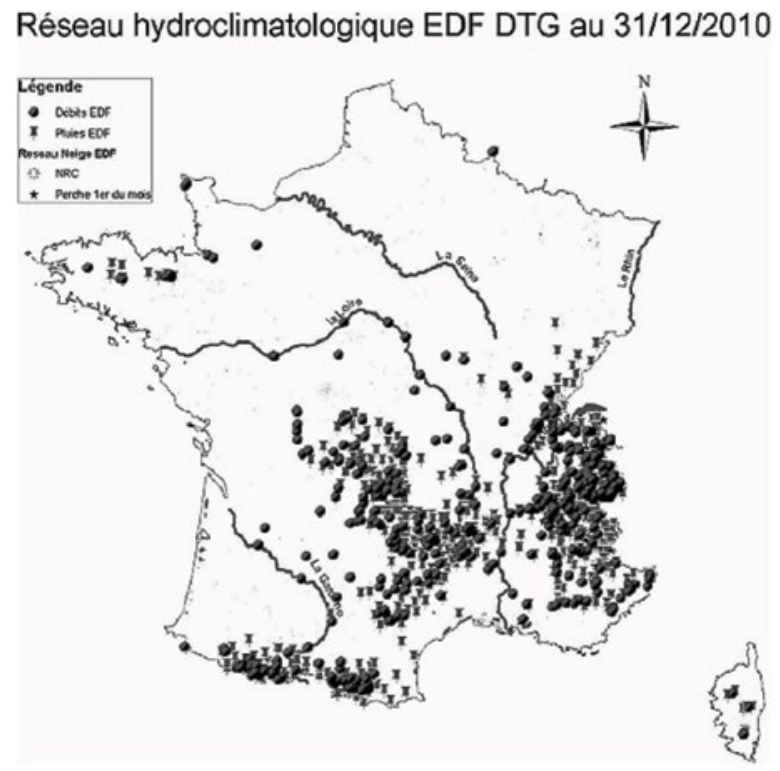

Figure 6 : Carte du réseau d'observation hydroclimatologique EDF en 2011 avec 840 points de mesure dont 340 pluviomètres, 180 mesures d'enneigement et 320 mesures de débit (Perret et al., 2012)

D. Duband mène le même combat à l'extérieur d'EDF pour que l'ensemble des gestionnaires de réseaux se dotent de moyens et de compétences suffisants au regard des objectifs de qualité à atteindre.

Il est ainsi à l'origine de la création du Comité Consultatif des Réseaux Climatologiques - le CCRC - qu'il présida de 1999 à 2004 et dont l'idée naquit des conclusions de l'Atelier 
sur « l'avenir des réseaux climatologiques » co-organisé à Toulouse en 1996 par le Ministère de l'Environnement, Météo-France et EDF.

Il s'agissait de réunir, autour de la table, les grands opérateurs de réseaux de mesure climatiques (Météo-France, EDF, les services du Ministère de l'Environnement, certains services d'annonce de crue...) et les utilisateurs de données climatologiques (laboratoires de recherche, bureaux d'études, collectivités territoriales, ...) pour aider à promouvoir des recommandations pour améliorer la qualité et la diffusion des données climatiques.

Le 20 décembre 2004, par arrêté du ministère des Transports auprès du Président Directeur Général de Météo-France, le CCRC devient officiellement le Comité Consultatif des Réseaux d'Observation Météorologiques ou CCROM dont D. Duband assura la première présidence. Cette nouvelle instance qui a repris en les élargissant les principales attributions et missions du CCRC, constitue aujourd'hui un lieu privilégié d'échanges et de concertation entre gestionnaires de réseaux et utilisateurs de données.

Le CCROM d'aujourd'hui doit beaucoup à la ténacité initiale de D. Duband à voir émerger une structure de ce type. Ainsi de 1996 à 1999, date de création du CCRC, il porta, chaque année, devant le Conseil Supérieur de la Météorologie (CSM) et jusqu'à satisfaction, un vœu demandant que soit «créé un Comité consultatif des utilisateurs de réseaux climatologiques comparable au Comité consultatif Aramis pour le réseau de radars météorologiques ».

Sur le modèle de ce CCROM, D. Duband a continué de s'investir jusqu'au bout pour que soit créé aussi un $\mathrm{CCROH}$, comité du même type pour les réseaux d'observations hydrométriques. A cette heure, ce comité n'existe toujours pas mais l'occasion nous est ainsi donné de continuer avec d'autres à militer pour sa création.

\section{LA DÉFENSE ET LA SAUVEGARDE DES DONNÉES ANCIENNES}

On ne saurait être complet ici, si on n'évoquait pas la contribution de D. Duband à la recherche et à la valorisation des données anciennes en hydrologie et en météorologie.

C'était, si l'on peut dire, son combat permanent qui allait de pair avec son regard de statisticien, grand adepte des chroniques les plus longues pour diminuer les effets d'échantillonnage, mais aussi avec sa conviction que toute mesure - pour autant qu'elle ait été correctement élaborée -, restait la meilleure représentation de la réalité des phénomènes et qu'elle devait, en cela, être toujours recherchée et précieusement sauvegardée.

Pour les données climatologiques, c'est surtout au travers de la Commission Hydrologie du Conseil Supérieur de la Météorologie (CSM) qu'il présida pendant quinze ans jusqu'en 2007 qu'il se fit le meilleur avocat de la récupération des longues séries anciennes de précipitations et de pression atmosphériques. Toujours au travers de vœux qu'il présenta devant le CSM - à l'instar de ce qu'il fit pour la création du CCRC (voir supra, § III) - il demanda ainsi, dès 1992, au travers du vœu ${ }^{\circ} 2$ que "la saisie des données des archives pluviométriques anciennes à Fontainebleau soit encouragée et organisée par Météo-France, soit (...) à travers les Centres Départementaux de la Météorologie (CDM) soit en collaboration avec les utilisateurs potentiels qui pourraient fournir personnel et moyens financiers alors que Météo-France apporterait savoir et méthodologie ».
Egalement en 1997 (vœu $\mathrm{n}^{\circ}$ 2) pour que « le projet concernant la constitution d'une banque de données de longues séries d'observations historiques soit poursuivi jusqu'à la critique des données ». Puis en 2001 (Vœu N 1) pour que «Météo-France réalise un livret sur l'état actuel des informations climatologiques anciennes, avec des précisions sur les types d'accessibilité, les services fournisseurs concernés (locaux, régionaux, nationaux), les modalités d'accès et les coûts des services" ».

Là encore, la ténacité et l'action continue de D. Duband pour valoriser les données anciennes trouva un épilogue favorable grâce à l'engagement en 2011 par Météo-France - qu'il convient ici de féliciter - du projet Triple A d'Accès aux Archives du climat de Fontainebleau en dépit de l'Amiante.

Il faut dire que le jeu en vaut la chandelle puisqu'on a là, en effet, $2 \mathrm{~km}$ linéaires d'archives avec plus de 6300 cartons, assez bien répertoriés et localisés. Ils contiennent, outre des données climatologiques, des fonds de prévisions - notamment des cartes -, et d'autres documents des anciens services de recherche de la Météorologie Nationale et de divers départements de la Direction générale (Figure 7, page suivante).

Hors les problèmes de désamiantage qui sont à résoudre car les données les plus intéressantes sont justement dans les parties amiantées, ces archives sont en très bon état de conservation. Cette action reconnue comme prioritaire au niveau des Archives Nationales et inscrite dans leur programme de travail triennal, a été soumise à une Fondation privée pour inventorier complètement le contenu des cartons, définir les priorités de saisie et engager le travail de numérisation des données les plus intéressantes. Elle est maintenant bien engagée et fait l'objet de restitutions régulières par Météo-France devant la Commission Hydrologie du CSM.

Cette action continue de D. Duband au service de la sauvegarde et de la valorisation des données anciennes, on la retrouve dans l'attention qu'il a toujours portée à la récupération de plusieurs fonds d'archives hydrologiques : les archives de M. Pardé qu'il contribua à sauver d'un déménagement universitaire avec l'aide de X. Martin (Huet, 2013) ou celles de J. De Beauregard ${ }^{7}$ dont je me vois encore, avec D. Duband, vider les armoires de son bureau à Paris pour remplir des cartons de documents et de tableaux de données que nous fîmes rapatrier à la DTG à Grenoble.

On la retrouve aussi dans les nombreux écrits ou articles qu'il a consacrés aux longues séries historiques de précipitations et de débits et au parti qu'il en a toujours tiré pour rappeler à chacun que le climat du futur est d'abord inscrit dans son passé le plus lointain. Chacun a en tête les multiples références qu'il faisait constamment aux étiages historiques sévères de la Loire à Blois, aux grandes crues du passé du bassin du Rhône ou à l'hydrologie et à la climatologie des grands fleuves européens et de leurs étiages les plus sévères (Duband et al., 2004).

Cet engouement pour les longues séries de données, il le devait bien sûr à sa formation de statisticien et à sa sensibilité toute particulière aux questions d'échantillonnage. Il le devait aussi à l'intérêt et à une forme de fascination qu'il avait pour tout ce qui touche au "climat » au sujet duquel il était intarissable mais aussi parfois " infranchissable ».

7. J. De Beauregard, ingénieur à la Direction de l'équipement d'EDF contribua, pendant de longues années, à la réalisation de l'Annuaire hydrologique de la France, les fameux annuaires verts de la SHF. 


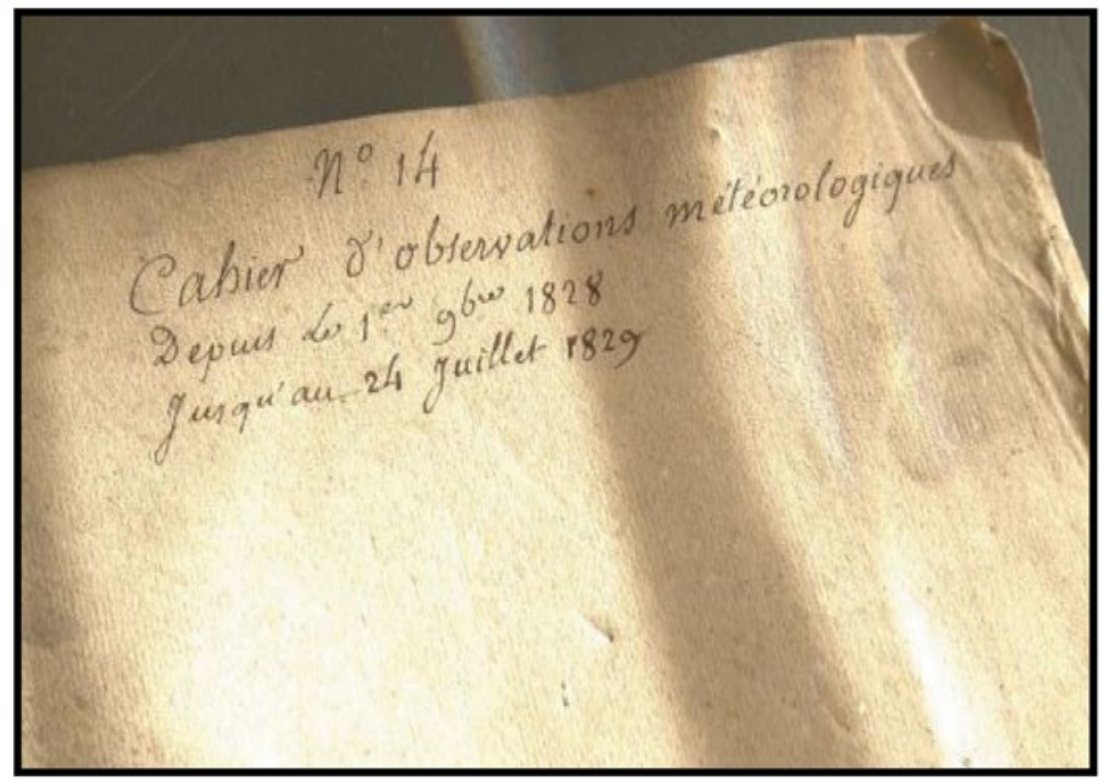

Figure 7 : Document extrait des premiers traitements des archives de Fontainebleau dans le cadre du Projet Triple A de Météo-France (Source Météo-France).

Ce rapport quasi passionnel qu'il avait sur les questions relatives au climat ou au changement climatique a marqué beaucoup d'entre nous. Qui ne se souvient pas, en effet, de ses refus d'interpréter une tendance dans une série comme les prémices d'un changement climatique déjà en route.

Très tôt, en 1989-90, alors que la France traverse une importante sécheresse (Duband et al., 1989) et que le premier rapport du GIECC vient juste de paraitre, il pose la question de savoir si l'on peut « discerner actuellement dans l'analyse des chroniques historiques de mesures climatologiques une perspective de changement de climat ?» (Duband, 1992). En remontant le plus loin dans le passé des longues séries de températures et de précipitations disponibles, il met en avant les « séquences » d'années particulières qu'il y décèle plutôt que des tendances irréversibles. Il pousse ainsi la communauté scientifique à bien analyser les longues chroniques du passé avant de tirer des conclusions trop rapides de quelques séries trop courtes pour bien représenter la « respiration » du climat. Il rappelle aussi au passage la nécessité de préserver les très longues séries de données climatiques tout en continuant à étoffer les réseaux de mesure existants et propose même de créer un "label de qualité " pour identifier les très longues séries historiques homogènes.

Pour être complet au chapitre de la valorisation des archives et des données anciennes et comme un juste retour des choses, il convient de signaler enfin le travail que nous avons entrepris avec M. Lang (Irstea) et D. Cœur (Acthys) pour récupérer et mettre en forme les archives personnelles de D. Duband. C'était une demande de ses enfants de voir ainsi préserver l'ensemble des données et de la documentation qu'il avait conservé dans son bureau. Qu'ils en soient ici chaleureusement remerciés.

Au total, ce ne sont pas moins de 72 boîtes de documents de toutes natures (Cœur et al. , 2013) qui ont été triées et référencées et qui seront versées demain dans un fonds d'archives publiques, probablement à la bibliothèque universitaire des sciences de Grenoble.

Il faudrait trop de temps pour décrire ici toute la richesse des documents ainsi sauvegardés mais, pour qui s'intéressera demain à l'hydrologie des crues et des étiages de nos cours d'eau (rubrique 2 du plan de classement), aux longues séries hydroclimatologiques de référence (rubriques 5) ou aux méthodes et outils scientifiques que D. Duband a développés (rubrique 2), il y a là un capital d'informations et de données qu'on ne saurait ignorer.

\section{L'APPUI AUX INSTANCES SCIENTIFIQUES ET INSTITUTIONNELLES DE L'HYDROLOGIE ET DE LA MÉTÉOROLOGIE}

Il serait fastidieux, ici, de lister toutes les instances, comités techniques, conseils scientifiques, commissions, missions d'évaluation, groupes de travail, comités d'organisation auxquels D. Duband a apporté sa contribution et qu'il a enrichis de ses questionnements et de ses propositions.

On a déjà évoqué plus avant sa contribution active à la Commission Hydrologie du Conseil Supérieur de la Météorologie. Il en assura la présidence pendant près de quinze ans et en fit son meilleur outil pour favoriser le rapprochement entre hydrologues et météorologues et faire en sorte avec persévérance et force exigence qu'hier la Météorologie Nationale, aujourd'hui Météo-France, réponde toujours présent aux demandes de la communauté hydrologique.

On a évoqué également son rôle dans la création du Comité Consultatif des Réseaux d'Observation Météorologiques (CCROM) qui est aujourd'hui un lieu privilégié d'échanges et de concertation entre gestionnaires de réseaux de mesures et utilisateurs de données.

D. Duband a été aussi rapporteur de nombreux dossiers au sein des Conseils Scientifiques des «Programmes de Recherche sur les inondations » initiés par le Ministre de l'environnement des années 1995-2005. Il est également intervenu, toujours bénévolement, en appui technique à la Mission interministérielle de l'Environnement (MISE) devenue Inspection Générale de l'Environnement - lors des 
missions de Retour d'expérience menées pour le compte de l'Etat, après plusieurs inondations catastrophiques.

En particulier, il est intervenu très efficacement lors des inondations du Gard de 2002, en animant un groupe d'hydrologues et de météorologues constitué pour l'occasion. Il retrouva là, comme déjà évoqué plus avant, une opportunité supplémentaire à faire se rapprocher hydrologues et météorologues et à les faire converger vers un corpus d'explication commun pour démythifier et replacer l'évènement dans son contexte méditerranéen global de l'Espagne à l'Italie.

Après la crue du Rhône de décembre 2003, il joue aussi un rôle déterminant dans l'expertise du débit maximal de la crue du fleuve à Beaucaire, objet d'une vive controverse entre les parties en présence et participe à la conférence de consensus qui sera organisée par le Préfet Coordonnateur du Bassin Rhône-Méditerranée du 26 au 28 juillet 2005 à la demande du Ministère de l'écologie. Sa contribution qu'il construit avec $\mathrm{Ph}$. Bois (Duband et Bois, 2005) autour d'une analyse très fine des jaugeages disponibles à Beaucaire et de tous les hydrogrammes de crue du Rhône et de ses affluents, sera une pièce déterminante pour arrêter à $11500 \mathrm{~m} 3 / \mathrm{s}$ l'estimation la plus vraisemblable de ce débit maximal et lui affecter une durée de retour légèrement supérieure à 100 ans.

On retrouve également sa marque dans de nombreux avis du Conseil scientifique du Comité de Bassin Rhône-Méditerranée où sa compétence et son expertise en hydrologie et hydroclimatologie sont d'un grand apport pour des dossiers à enjeux aussi divers que la liaison Rhin-Rhône, le projet de transfert d'eau de Nîmes à Barcelone, le Plan Rhône de protection contre les inondations ou l'étude de l'impact des rejets thermiques des centrales nucléaires sur la température du fleuve.

Enfin, sa contribution, pendant toute sa carrière, à la vie de la Société Hydrotechnique de France (SHF) comme membre du Comité Scientifique et Technique et surtout comme président de la Division Eau Environnement mérite d'être soulignée tout particulièrement. Lorsqu'il était encore en activité à la DTG, il a aussi co-organisé pendant de longues années, les journées de glaciologie-nivologie de la SHF, rendez-vous annuel obligé de tous ceux qui s'intéressent en France, en Suisse, en Italie et en Espagne aux questions relatives à la climatologie de la neige et des glaciers de haute-montagne.

Parcourir les thèmes de tous les colloques SHF qu'il a montés et organisés au cours des 20 dernières années et en reprendre les grands débats, serait nécessairement fastidieux. Disons simplement qu'on y retrouve tous les grands axes que nous avons évoqués dans ce document - la prévision opérationnelle, la critique des données, la défense des réseaux de mesure, la valorisation des séries anciennes, les évènements extrêmes, le climat et sa variabilité, ... - et les thèmes scientifiques que Ch. Obled (2013) évoque de son côté dans son papier.

Avec, in fine, toujours la volonté qui fut sienne de faire progresser la communauté des hydrologues et des météorologues vers une hydrométéorologie opérationnelle de qualité et au service des enjeux de tous.

\section{Remerciements}

Je tiens à remercier tous ceux qui, par leurs remarques ou suggestions, ont contribué à enrichir cet hommage : R. Garçon et Ch. Perret qui restent à la DTG les continuateurs de l'esprit que D. Duband a su imprimer à l'hydrométéorologie opérationnelle d'EDF ; J. Pinte, comme moi, ancien et fidèle collaborateur de D. Duband; Ch. Obled, professeur émérite à l'INP de Grenoble qui rend un hommage très complet (Obled, 2013) à la contribution scientifique de D. Duband ; également Ph. Huet qui a souvent fait appel à lui lorsqu'il était à l'Inspection générale de l'environnement et au sein de l'AFPCN ; B. Chastan qui l'a côtoyé au sein du Conseil Scientifique du Comité de Bassin Rhône-Méditerranée ; enfin, M. Lang de l'Irstea qui a pris sa suite, au sein de la SHF et avec qui, aux côtés de D. Cœur (Acthys), nous avons travaillé à la récupération et à la mise en forme des archives personnelles de D. Duband.

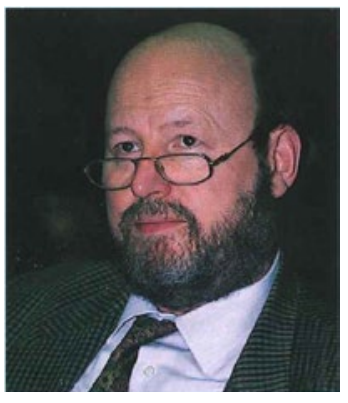

D. Duband, photo EDF

\section{RÉFÉRENCES}

Ben Daoud A. (2010) - Améliorations et développements d'une méthode de prévision probabiliste des pluies par analogie. Application à la prévision hydrologique sur les grands bassins fluviaux de la Saône et de la Seine - Thèse Université Grenoble

Bontron G. (2004) - Prévision quantitative des précipitations : adaptation probabiliste par recherche d'analogues. Utilisation des réanalyses NCEP/NCAR et application aux précipitations du sud-est de la France - Thèse INP-Grenoble

CAstaing M. (1993) — Des spécialistes préconisent la création d'un organisme de prévention unique. Le Monde. 9 octobre 1993

Ceeur D., Lang M., Tourasse P. (2013) - Inventaire du fond d'archives personnelles de Daniel Duband - Document de travail, 13 septembre 2013. 3

Deneau V., Leprieur C., Saint G. (1981) — Télédétection de la surface enneigée sur les plateaux du Massif-Central. Journées SHF de Glaciologie des 12 et 13 mars $1981.12 \mathrm{p}$

DENEAU V. (1981) - L'accumulation de neige sur les lignes électriques - Les incidents du 11 janvier 1981. Rapport d'étude EDF-DTG - 19 octobre 1981. 30 pages et annexes

Deneau V., Guillot P. (1984) — La neige collante - Essai de cartographie du risque sur l'ensemble de la France. Journées SHF de Glaciologie des 14 et 15 mars 1984. 17 pages et annexes

DuBAND D. (1970) - Prévision d'apports de la Loire à Gien en période d'étiage (juin à octobre) - Rapport d'étude DTG Décembre 1970. 60 pages et annexes

DuBAND D. (1970) - Reconnaissance dynamique de la forme des situations météorologiques - Application à la prévision quantitative des précipitations. Thèse de la Faculté des sciences de Paris

Duband D. (1973) - Neige collante pour le Centre Régional de Transport du Sud-Ouest - Note Edf-DTG, 26 août 1973. 14 pages et tableaux.

Duband D. (1980) - Rationalisation d'un réseau de mesure de la précipitation. Revue La Météorologie. Numéro spécial, Juin 1980. 219-231

Duband D. (1981) - Choix d'emplacements représentatifs de l'enneigement dans les Alpes du Nord. SHF. Section de Glaciologie. Grenoble Saint Martin d'Hères, Actes du colloque, mars 1981

Duband D., Deneau V. (1982) - Prévision journalière de neige collante et de givre - Note EDF-DTG, 5 août 1982

Duband D., Michel C., Garros H. Et Astier J. (1988) Evaluation des Crues Extrêmes et de la Crue de Projet par la Méthode du Gradex. Estimating Extreme value Floods and the Design Flood by the Gradex Method. Commission Internationale des Grands Barrages, XVI ème Congrès des grands Barrages, San Francisco, Q 63-R60. IV 1009-1047 
DuBAND D. (1988) - Bilan de 35 ans de mesure de neige, hauteur, densité, valeur en eau effectuées par EDF en haute et moyenne montagne. Journées SHF de Glaciologie des 10 et 11 mars 1988. $14 \mathrm{p}$

Duband D., Tourasse P., Pinte J. (1989) - Le déficit des précipitations dans le sud de la France (pluie-neige) pendant l'hiver 1988-1989 : conséquences sur l'hydrologie de haute et moyenne montagne. La Houille Blanche. 7-8 534-543

DuBAND D. (1992) - Peut-on discerner actuellement dans l'analyse des chroniques historiques de mesures climatologiques une perspective de changement climatique. XXIIèmes journées de l'Hydraulique - L'avenir de l'eau, Paris, 15-17 septembre 1992. $12 \mathrm{p}$

DuBAND D. (1994) - Estimation des crues extrêmes de la rivière Saint-Maurice à la Tuque et à la Gabelle par la méthode du Gradex, Note EDF-DTG du 23 novembre 1994. $16 \mathrm{p}$

Duband D., Schoeneich Ph., Stanescu V.A. (2004) - Exemple de l'étiage 1921 en Europe (Italie, France, Roumanie, Suisse, ...) : climatologie et hydrologie. La Houille Blanche. 5 18-29

Duband D., Bois PH. (2005) - Rapport sur la crue du Rhône et ses affluents du ler au 5 décembre 2003 - Conférence du consensus du 26 au 28 juillet 2005. 40 p

Duband D., Obled Ch., Rodriguez J.Y. (1993) - Unit Hydrograph Revisited : An Alternate Iterative Approach for U.H. and Effective Precipitation Identification. J. of Hydrology. 150 $115-149$

EDF-DTG (2006) - Synthèse de l'étude thermique Rhône - Note EDF-DTG de synthèse de l'étude de l'impact cumulé des rejets thermiques des CNPE le long du Rhône, 25 avril 2006

GARÇON R. (1988) - Evaluation des crues extrêmes du Sinnamary au barrage de Petit-Saut - Rapport d'études DTG

GARÇON R. (1996) - Prévision opérationnelle des apports de la Durance à Serre-Ponçon à l'aide du modèle MORDOR. Bilan de l'année 1994-1995. La Houille Blanche 5 71-76

Garçon R., Houdant B., Garavaglia F., Mathevet Th., Paquet E., GAIllard J. (2009) - Expertise humaine des prévisions hydrométéorologiques et communication de leurs incertitudes dans un contexte décisionnel. La Houille Blanche. 5 71-80

GT THERMie (2012) - Les rejets thermiques des centrales nucléaires en période de canicule - Document de synthèse ASN-MEEDD-EDF du Groupe de Travail "Rejets Thermiques ", 10 septembre 2012.7 pages

Guilbaud S. (1997) - Prévision Quantitative des Précipitations Journalières par une méthode Statistico-Dynamique de Recherche d'Analogues. Application à des Bassins du Pourtour Méditerranée - Thèse INP-Grenoble. 386 pages

Guillot P. Et D. Duband (1980) — Une méthode de transfert pluie-débit par régression multiple. Oxford Symposium on
Hydrological forecasting - Prévisions hydrologiques, April. Actes du Colloque d'Oxford, IAHS-AISH Publ. 129 p. 177-184

Horton P. (2012) — Améliorations et optimisation globale de la méthode des analogues pour la prévision statistique des précipitations. Développement d'un outil de prévision et application opérationnelle au bassin du Rhône à l'amont du Léman - Thèse de l'Université de Lausanne, Faculté des géosciences et de l'environnement

HuEt PH. (2013) - Le rôle d'appui technique de Daniel Duband aux pouvoirs publics et aux associations - Note personnelle à l'auteur. 2 pages

LAPEYRE J.-L. (1990) — La neige collante et les lignes aériennes. Numéro spécial de La Recherche pour EDF - DER. XXIX-XXXI

Maurin G. (1995) - Histoire du Service de la Production Hydraulique (SPH) d'EDF - 1946-1992 - Ouvrage collectif publié par l'AHEF (Association pour l'histoire de l'électricité en France) sous la Direction de G. Maurin. 640 pages

Obled CH. (2014) - Daniel Duband : 50 ans de contributions scientifiques à 1'hydrologie statistique (1962-2011). La Houille Blanche. 2 xxxxx

Paquet E., Garavaglia F., Garcon R., Gailhard J. (2013) - The SCHADEX method: a semi-continuous rainfall-runoff simulation for extreme flood estimation. J. Hydrology. 495 23-37

Perret CH. (1992) - Retour d'expérience du fonctionnement du réseau hydroclimatologique temps réel d'EDF lors de l'épisode du 21 septembre 1992. Note technique EDF-DTG

Perret Ch., Hauet A, Parrel D., Saysset G., Vignon Ph., SchNeGg P. (2012) - Le réseau hydroclimatologique de montagne EDF - Etat des lieux. La Houille Blanche. 3 18-25

Pinte J. (1981) - Choix d'emplacements représentatifs de l'enneigement dans les Alpes du Nord, Rapport EDF-DTG, 17 mars 1981

Pinte J., Berrut F. (1986) - Synthèse de l'épisode neigeux des 30 et 31 janvier 1986 sur les Pyrénées et le Roussillon, Note EDF-DTG, 28 mars 1986. $25 \mathrm{p}$

Plancher C. (1976) - Evaluation du risque de formation de neige collante sur les lignes du sud-Est, Note EDF-DTG, 26 mai 1976. $24 \mathrm{p}$

Poirel A. (1997) - Evolution interannuelle des températures de l'eau - Liaison avec les températures de l'air, incidence sur les études biologiques. Note EDF-DTG, D4161 - DTG RE ENV 97-053-A

Tourasse P., Drouet V. (1990) - Prévision et suivi de l'épisode de neige collante du 9 décembre 1990. Note EDF-DTG, 13 décembre 1990. $13 \mathrm{p}$

Tourasse P. (1991) - Evaluation des crues extrêmes de la Yaté au barrage de Yaté (Nouvelle-Calédonie). Rapport EDF-DTG. $130 \mathrm{p}$ 